Alejandro Crispiani

\title{
Un mundo continuo
}
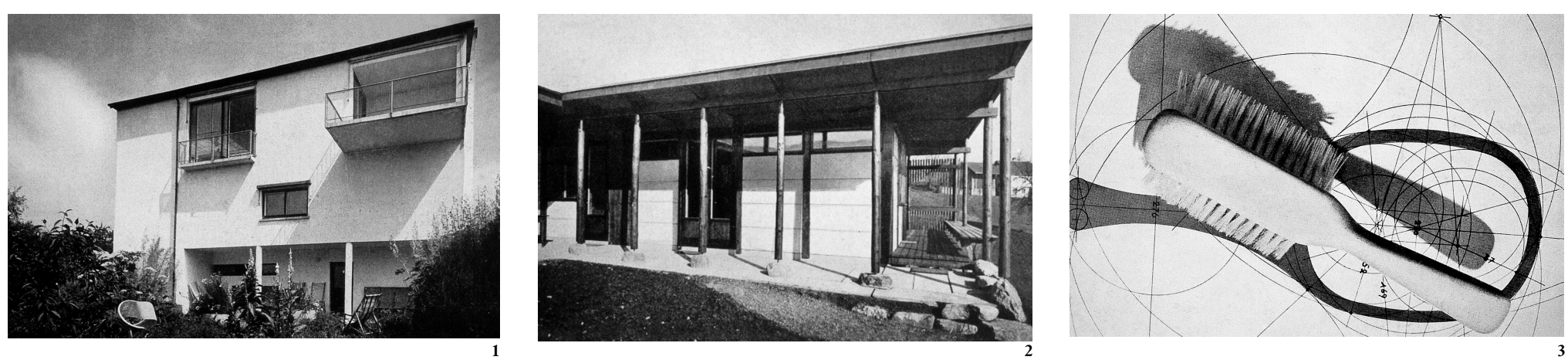

La propagación del lema "diseñar de la cuchara a la ciudad" tuvo su momento de auge entre los años ' 40 y' 60 y su irradiación por América Latina fue rápida, con una gravitación que se prolongó hasta fines de los '70.

De todas las figuras que se identificaron con este lema, la que más acabadamente lo encarnó, fue el artista y diseñador suizo Max Bill. Crissiani nos entrega una visión del trabajo de Bill a partir de un análisis de las formulaciones que el suizo desarrolla en torno al "arte concreto" y la "buena forma", entre otras.

The dissemination of the motto "design from the tablespoon to the city" had its peak moment around the 40's and 60's and its irradiation to Latin America was quick, with a gravitation that lasted until the end of the 70's

Among the figures that were identified with this motto, the one that more completely embodied it was the artist and Swiss designer Max Bill. Crispiani gives us a vision of Bill's work starting from an analysis of the concepts that the Swiss developed around the "concrete art" and the "good form", among others.

Aún resta por hacer un estudio profundo de las consecuencias y la importancia que el antiguo lema "diseñar de la cuchara a la ciudad" tuvo para la cultura arquitectónica y de diseño en América Latina. Hoy en día este lema, detrás del cual se condensaba una larga ambición vanguardista y al que se asociaba una galería bastante precisa de nombres, no puede menos que parecer lejano e ingenuamente ambicioso. Su atractivo se ha desvanecido por completo y su falta de asidero en la realidad concreta de la producción de arquitectura, ciudad y diseño ha quedado hace tiempo al desnudo. Es un cul de sac de la teoría del diseño y la arquitectura. Uno de los tantos escombros de la historia, podría pensarse. Sin embargo, no debería menospreciarse la importancia de estos intentos aparentemente fallidos: gran parte del tejido de la historia de la cultura, cuando se lo mira de cerca, parece estar constituido por estas aparentes "vías muertas", por caminos muchas veces sin resolución final ni continuidad explícita con el presente.
"Diseñar de la cuchara a la ciudad" fue un programa y una fantasía, y pocas cosas resultan más embarazosas que las fantasías pasadas e irrealizables. Tuvo su momento de auge en los años posteriores a la segunda posguerra, entre finales de los años cuarenta y los sesenta, y un origen bastante preciso: la reconstrucción de Alemania Occidental empujada por el Plan Marshall. Su irradiación en América Latina, particularmente en el cono Sur, fue rápida y su gravitación se prolongó, por distintos medios, hasta casi los años setenta. Muchas fueron, aparentemente, sus ramificaciones. Baste señalar que en las dos décadas posteriores a la Segunda Guerra Mundial, cuando las primeras manifestaciones del diseño moderno comienzan a emerger y en algunos casos a tomar forma institucional en América Latina, la constelación de ideas que encierra el programa ya citado era uno de los horizontes teóricos más atractivos, que surgía como la prolongación en algunos casos superadora de los postulados modernistas de cuño Bauhaus, manteniendo sobre nuevas bases su visión comprensiva (aunque no integradora) de las disciplinas proyectuales.

Como es bien sabido, de todas las figuras que en la segunda postguerra se identificaron con este programa, la que más acabadamente lo encarnó en su pensamiento y en su producción, siendo casi la personificación del mismo, fue el artista y diseñador suizo Max Bill. Primer director de la Hochschule fur Gestaltung de Ulm, entre 1947 y 1955, Max Bill estuvo en el centro de dos movimientos complementarios en el campo del arte y del diseño, cuyos derroteros terminaron separándose con el correr de la segunda mitad del siglo XX pero que obedecieron en su momento a un mismo impulso: el Arte Concreto y las teorías de la Buena Forma (Gute Form). Ambas eran, evidentemente, una decantación y actualización de un cierto segmento de los

$\begin{array}{lc}1 \text { Casa Bill (vista desde el } & \text { 2 Casa en Bremgarten, } 1942 . \\ \text { jardín). Zürich, 1932. Fuente: } & \text { Ibídem, pág. 108 } \\ \text { Maldonado, Tomás. Max Bill, } & \text { 3 Cepillo para el cabello, } \\ \text { Nueva Visión, Buenos Aires, } & \text { 1948-49. Ibídem, pág. 122 } \\ \text { 1955; pág. 105 } & \end{array}$

1955; pág. 105 postulados vanguardistas de los años veinte. Ambos eran, además, un intento por restablecer las conexiones del arte y del diseñño con el campo de la ciencia y de la tecnología, basándose en la búsqueda de una racionalidad que les fuera propia. Frente a la irracionalidad del styling $y$ de las corrientes del arte informal de la segunda posguerra, y frente a los embates de las vanguardias norteamericanas, las teorías de la Buena Forma y del Arte Concreto aparecieron como la contestación constructiva, y en gran medida europea, a estos intentos. Contestación, como puede verse, que iba más allá de adjudicarse el dominio de la ideación del más pequeño utensilio al más complejo de los centros urbanos, para proyectarse a todos los productos materiales de la cultura, entre los cuales se encuentran, obviamente, las obras de las artes plásticas.

Las teorías de Bill parten de la aceptación de que en el inmenso abanico de los productos materiales de una cultura existen jerarquías, funciones y niveles de operación completamente distintos pero que pueden, en algúún punto, no sólo armonizarse sino formar un entramado coherente de actividades y conocimientos. Para comprender cómo fue pensada la posibilidad de arribar a este entramado es necesario detenerse, aunque sólo sea brevemente, en la formulación que hace Bill tanto del Arte Concreto como de la Buena Forma.

El Arte Concreto fue para Bill la idea matriz que orientó sus teorías y sobre la cual se fundó toda su producción artística. El concepto de lo concreto en el arte, en realidad, ya había sido formulado por Theo van Doesburg a principios de los años. Se basaba, en principio, en el reconocimiento y en la definición de los materiales constitutivos esenciales de cada una de las expresiones artísticas. En el caso de la música, por ejemplo, estos materiales eran, evidentemente, los sonidos, pero todos los sonidos, no sólo aquellos producidos 

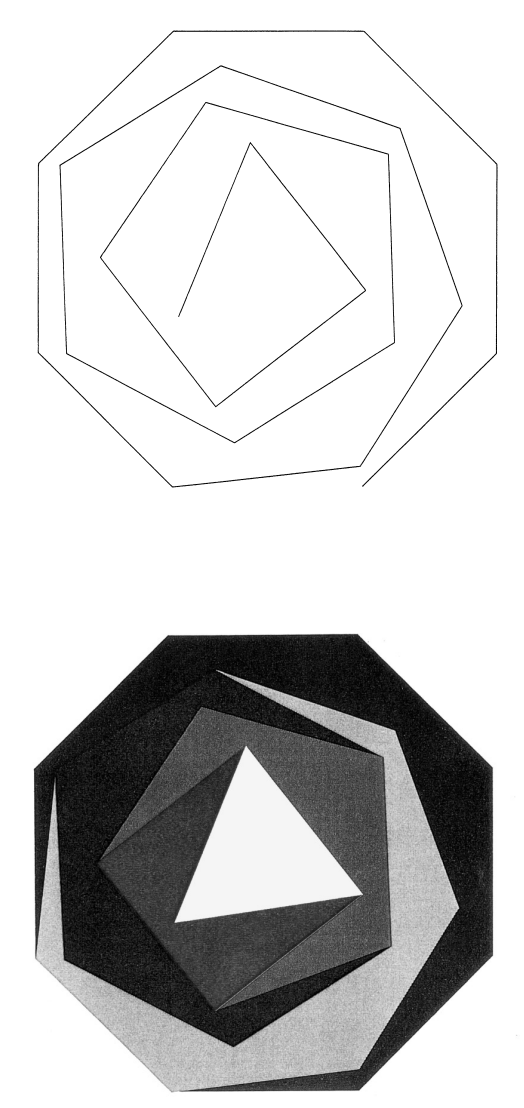
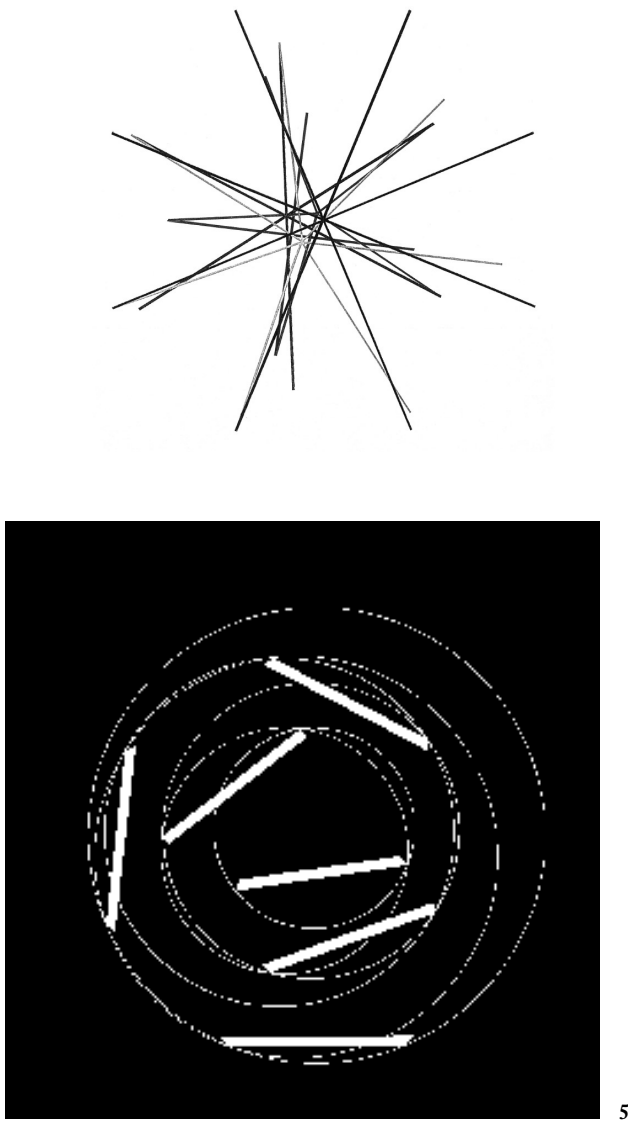

4 Variación $\mathrm{N}^{\circ} 1$. Fuente: Helmhaus Zürich. Max Bill, Zürich, 1984; pág. 30

5 Variación $\mathrm{N}^{\circ}$ 5. Ibídem, pág. 32

6 Variación $\mathrm{N}^{\circ} 10$. Ibídem, pág. 35
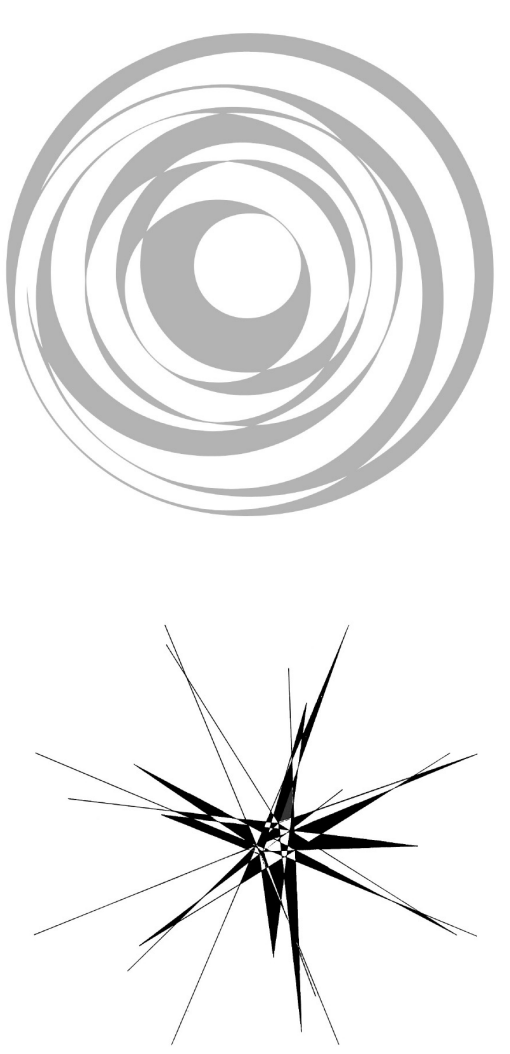

por los instrumentos. En el caso de la pintura, estos materiales constitutivos eran la línea, la superficie y el color. Se trataba de operar con estos elementos de manera tal de producir una "realidad nueva", que no intentara representar o reproducir nada "existente", sino explorar la propia lógica de asociación que estos elementos demandaban según un proceso verificable $y$ racional. En tal sentido, el arte concreto se oponía al arte abstracto (del que se suponía un desprendimiento), ya que no era el resultado de ninguna abstracción de una realidad preexistente, sino que construía su propia realidad según las leyes de asociación que sus elementos constitutivos le dictaban. En el presupuesto que estas leyes debían ser del mayor rigor y exactitud, no es extraño que Bill se volviera hacia las matemáticas, y en particular hacia la topología, en busca de inspiración y de principios. Así como las matemáticas habían logrado crear su propio e insondable universo de problemas a partir del concepto de número, así deberían proceder las artes plásticas a partir de los conceptos que les fueran propios. No se trata de aplicar las matemáticas a las artes plásticas (aunque algunos cuadros de Bill parecen caer en esta posibilidad) sino de construir una realidad con el mismo grado de especificidad y rigor metodológico. Pero esta especificidad y autonomía no implicaría justamente aislamiento sino todo lo contrario.
Es justamente desde su autonomía que las matemáticas pueden constituirse, según Bill, en el fundamento de las ciencias exactas y, desde ellas, de las tecnologías. Una relación análoga es la que intenta crear Bill entre las artes plásticas y las disciplinas proyectuales: las matemáticas serían a la física y a la química, por ejemplo, lo que las artes plásticas serían a la arquitectura y el diseño. Se trata de lo que un crítico llamó la "función blanca del arte", entendido como el campo donde lo absoluto se pone en juego.

Las ideas de la "buena forma" descansan sobre esta base y son en gran medida el puente que une al campo del arte con las disciplinas proyectuales. La definición de forma de la cual parte Bill es simple, casi rudimentaria, pero efectiva: "forma es

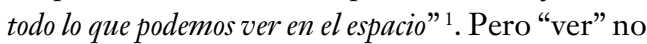
es reconocer. Ver significa comprender, significa percibir algo como inteligible a partir del sentido de la vista. Por eso la forma se presenta como una cualidad: hay objetos que tienen más forma y objetos que tienen menos forma. La manera en que el objeto se hace inteligible y brinda esta inteligibilidad a la vista es el punto central de la "buena forma", que vale tanto para las obras artísticas como para los productos de las disciplinas proyectuales. En el caso de una pintura, por ejemplo, esta inteligibilidad podría lograrse a partir de la utilización de una cierta proposición matemática, de la cual el cuadro se piensa como una especie de visualización o de recreación desde el punto de vista de la sola utilización de líneas, colores y superficies. ¿Pero en qué radicaría, concretamente, el "más forma" cuando se halla referido a los objetos de uso o la arquitectura? ¿Qué sería lo que tienen que hacer inteligible? Dos serían los puntos más evidentes: en primer lugar, el hacerse de cada objeto, el proceso por medio del cual se han constituido, vale decir, la tecnología que los ha hecho posibles. En segundo lugar, el uso o los usos al que están destinados. Ambas cuestiones, que Bill entiende como funciones del objeto, deberían ser compatibilizadas justamente por la "forma". La buena forma sería aquella que no sólo logra hacer inteligible al objeto en términos de uso y tecnología, sino que, además, logra mostrarse como la armonización de ambos factores, es decir, logra exponerse a la vista y al pensamiento como una unidad de funciones.

Esta postura, justamente, que pone a la cuestión de la forma en un primer plano y que hace del arte el ámbito en el que con mayor pureza pueden estudiarse sus leyes, va a ser rechazada en el interior de la Escuela de Ulm por distintos profesores, llevando al alejamiento de Bill de la dirección de la misma en 1955. Lo que van a esgrimir los críticos al programa de Bill, entre los que se encontraban varios de los futuros directores de la Escuela, como Tomás Maldonado 
y Otl Aicher, es la irrelevancia que, frente a los dramáticos progresos tecnológicos y científicos realizados en la segunda postguerra, supone el plantearse el diseño en términos de "forma" o relación con el arte.

Evidentemente, el programa de Max Bill se hallaba anclado en los problemas del período de entreguerras y de la inmediata postguerra. $\mathrm{Su}$ producción de objetos de diseño de los años cincuenta, por ejemplo, hacía referencia a un mundo industrial que en 1955 ya había sido superado. Cuestiones como la absoluta repetibilidad de las partes o la economía de materiales, que habían sido centrales, por ejemplo, en el diseño Bauhaus, habían pasado a ser, según los críticos al programa de Bill, criterios de segundo orden en el momento de diseñar un objeto. La tecnología se había demostrado mucho más flexible y los criterios de economía mucho más complejos que en los momentos iniciales del diseño moderno.

Sin embargo, las tesis de Bill implican un enfoque original a la cuestión de la relación entre las distintas disciplinas proyectuales, y de éstas con el campo de las artes plásticas, que va a tener múltiples derivaciones. Este juego de relaciones se constituye en torno a un concepto clave: el de continuidad de la experiencia estética. Esta idea no fue explícitamente formulada por Bill, pero se desprende de su pensamiento y de su producción, $\mathrm{y}$ de hecho fue puntualizada en su momento por algunos críticos ${ }^{2}$.

La idea de continuidad implica una doble dicotomía. En primer lugar, implica la existencia de distintas partes o más bien momentos, diferenciados entre sí e irreductibles a una simple unidad. Implica una conexión en la separación. Lo continuo no es simplemente lo aledaño, ni implica, tampoco, una integración. La continuidad es una alternativa tanto a la disgregación como a la integración. Supone que cada una de las disciplinas ha de obedecer a un grupo de problemas de la misma naturaleza, con un arsenal específico de herramientas y en, última instancia, de "elementos originarios". La teoría de la buena forma, con su énfasis en la justeza y la economía de los medios, su énfasis en lo visual y en los problemas del espacio, y su mandato de que toda actividad artística o proyectual debía tender a generar realidades en sí mismas, sin impurezas de otras realidades, era el principio que justamente permitiría el tránsito entre cada una de las manifestaciones de las disciplinas proyectuales y las artes plásticas.

En ese sentido, la propuesta teórica de Bill y también la que se desprende de su producción artística y de diseño, se diferencia claramente de la idea de integración de las artes, que con renovado vigor iba a surgir en la segunda postguerra. La integración, según se postulaba en los años cincuenta y como puede verse, por ejemplo, en distintas obras de Niemeyer, implica la concurrencia de las artes plásticas, la arquitectura y asimismo el diseño, hacia un mismo punto. Implica el superponerse de los conocimientos y de las técnicas en pos de una experiencia estética, podríamos decir, unificada. El muro entero puede ser un cuadro, y los elementos de arquitectura pueden entenderse en términos de escultura o viceversa. La continuidad que se postula desde el arte concreto niega esta posibilidad. Los elementos y los problemas de cada una de las artes o disciplinas proyectuales le son específicos. La diferencia entre ellas no es "histórica" sino "esencial". En tal sentido, un artista concreto como Tomás Maldonado podía reivindicar al cuadro, a la pintura en última instancia de caballete, como el verdadero tema de la pintura. No porque estuviera avalado por una larga tradición cultural, sino porque el cuadro corresponde con un cierto campo de visión dominable por la vista, campo de visión que permite comprender como una unidad los elementos pictóricos sin que se pierda su particularidad. Un muro nunca debería pensarse como un cuadro. El plano de un cuadro concreto es un absoluto, un muro nunca puede serlo. Nuevamente, es este volver a lo fundante y a lo irreductiblemente particular de cada una de las disciplinas proyectuales o artísticas, lo que garantizaría la continuidad entre ellas.

Por otra parte, continuidad implica también movimiento y cambio dentro de una cierta pauta de orden inamovible. El principio de la buena forma es, en tal sentido, un principio fluido, que no intenta una unidad formal explícita y claramente reconocible en los objetos a los cuales se aplica, pero que implica una cierta coherencia entre ellos, coherencia que es tanto visible como comprensible. O quizás sobre todo, comprensible. Un objeto de diseño, por ejemplo, debería revelar claramente su razón de ser y sus posibilidades de uso, la lógica económica y tecnológica que ha guiado su fabricación y el sentido de la operación formal que se revela a la vista. De la misma manera, una pintura de arte concreto debería plantear con evidencia cuál es el problema que intenta explorar en términos de relaciones de colores, superficies y líneas, problema que puede ser matemático (como es el caso de gran parte de la obra pictórica de Bill) pero que no excluye otras alternativas en la búsqueda de un principio aprehensible que conduzca la operación formal. Lo que se plantea, en última instancia, es un mundo variado pero sin rupturas, sin discontinuidades. Un mundo, como las pinturas en serie de Bill, donde cada cuadro completa y prolonga indefinidamente el razonamiento planteado por el anterior

Un mundo donde cada objeto se piensa como un tema de conocimiento, como un ejercicio de razonamiento y de rigor formal. La obra gráfica, las esculturas, los objetos de uso cotidiano, los proyectos de arquitectura, las pinturas y aún los monumentos urbanos de Max Bill podían mostrarse como prueba palpable y visible de esta posibilidad.

Y también de una aspiración más elemental y probablemente ingenua: la continuidad (no el reflejo condicionado o la reflexión post-facto) entre pensamiento discursivo y obra.

Alejandro Crispiani Enríquez

Arquitecto Universidad de La Plata, Argentina, 1984. Candidato a Doctor por la Facultad de Filosofía y Letras de la Universidad de Buenos Aires con la tesis "La trayectoria de la buena forma: del Arte concreto a las teorías del diseño para la periferia". Actualmente es profesor de la Escuela de Arquitectura de la Pontificia Universidad Católica de Chile. 